Fitness Effects of Floral Plasticity and Thermoregulation in a Thermally Changing

Environment

By: Elizabeth P. Lacey, Mary E. Lovin, Scott J. Richter

Lacey, E. P., Lovin, M. E., Richter, S. J. (2012). Fitness Effects of Thermoregulation in a Thermally Changing Environment. The American Naturalist, 180(3), 342-353. doi:

10.1086/666987

Made available courtesy of University of Chicago Press:

http://www.dx.doi.org/10.1086/666987

***@ $\subseteq$ University of Chicago Press. Reprinted with permission. No further reproduction is authorized without written permission from University Chicago Press.***

***Note: Full text of article below 


\title{
Fitness Effects of Floral Plasticity and Thermoregulation in a Thermally Changing Environment
}

\author{
Elizabeth P. Lacey, ${ }^{1, \star}$ Mary E. Lovin, ${ }^{1}$ and Scott J. Richter ${ }^{2}$ \\ 1. Department of Biology, University of North Carolina, Greensboro, North Carolina 27402; 2. Department of Mathematics and \\ Statistics, University of North Carolina, Greensboro, North Carolina 27402 \\ Submitted August 5, 2011; Accepted May 10, 2012; Electronically published July 19, 2012 \\ Online enhancement: appendix figures. Dryad data: http://dx.doi.org/10.5061/dryad.n872j.
}

ABSTRACT: To better understand the evolution of phenotypic plasticity and thermoregulation and their potential value for ectotherms in the face of global warming, we conducted field experiments to measure their effects on fitness and their association with reproductive phenology in Plantago lanceolata in a thermally variable environment. We measured the reproductive timing and success of genotypes varying in thermoregulation, as mediated by floral-reflectance plasticity. Results were consistent with the hypothesis that thermoregulation is more adaptive when thermally variable reproductive seasons are shorter and cooler. Strong thermoregulation/plasticity increased reproductive success during the cool portion of the reproductive season but not during the warm portion. Directional selection that favored strongly thermoregulating genotypes early in the season shifted to stabilizing selection that favored genotypes with weaker thermoregulation later in the season. Thermoregulation and reproductive phenology were negatively correlated. Although reproductive onset and duration were similar between genotypes, strong thermoregulators produced more and larger spikes (clutches) early; weak thermoregulators produced more spikes late. Results suggest that with atmospheric warming, the benefit of raising body temperature via thermoregulation when it is cool should decline in extant populations. The negative correlation between thermoregulation and phenology should accelerate the evolutionary shift toward thermoconformity, that is, reduced plasticity.

Keywords: fitness, thermoregulation, phenotypic plasticity, phenology, global warming, reproduction, Plantago lanceolata, phenotypicselection analysis.

\section{Introduction}

Near-surface atmospheric temperatures are predicted to rise between $2^{\circ}$ and $7^{\circ} \mathrm{C}$ in the twenty-first century (IPCC 2007), and ectotherms are expected to respond strongly to this change (e.g., Deutsch et al. 2008). Thermoregulation might be able to help ectotherms buffer the effect of this change. This ability allows an individual to modify its

\footnotetext{
* Corresponding author; e-mail: eplacey@uncg.edu.
}

Am. Nat. 2012. Vol. 180, pp. 342-353. (c) 2012 by The University of Chicago. 0003-0147/2012/18003-53238\$15.00. All rights reserved. DOI: $10.1086 / 666987$ physiology, morphology, and/or behavior in response to the external temperature to maintain an internal body temperature close to optimum. Whether or not thermoregulation will, in fact, facilitate the long-term survival of ectotherms is largely unknown because of limited information about the thermal plasticity, that is, the temperature sensitivity, of traits underlying thermoregulation in most ectotherms and about the potential contribution of this plasticity to local adaptation and dispersal (Helmuth et al. 2005; Kearney and Porter 2009; Kearney et al. 2009; Chevin et al. 2010; Chown et al. 2010; Gunderson et al. 2010; Hofmann and Todgham 2010; Wilczek et al. 2010). Current challenges are (1) to characterize the thermal plasticity of the underlying traits, (2) to independently estimate the fitness effects of this plasticity across natural thermal gradients, and (3) to clarify the interactions between underlying traits and other potential targets of selection imposed by climate change (Angilletta et al. 2006; Knies et al. 2009).

Here we present the results of three experiments that address these challenges. Our experiments focused on the evolution of thermoregulation in Plantago lanceolata L. (ribwort plaintain), a widespread temperate weedy herb. Plantago lanceolata partially thermoregulates its reproduction by changing floral reflectance (including color) in response to ambient temperature (Lacey and Herr 2005). Flowers are produced in tightly packed inflorescences, called spikes, produced throughout the reproductive season (e.g., mid-April-September in the Piedmont region of North Carolina). The process is analogous to the production of multiple clutches in animals. The reflectance of flowers in a spike is fixed by ambient temperature at the time of spike development. However, the reflectance of a spike and its flowers is reversible at the individual-plant level through the production of new spikes with different levels of reflectance. A $7^{\circ} \mathrm{C}$ temperature change in either direction produces a visible color change in new spikes relative to old spikes within a week (Lacey and Herr 2005). 
This temperature-sensitive plasticity in floral/spike reflectance is particularly high in the visible and near-infrared regions of the spectrum (fig. A1, available online; note the peak at $550 \mathrm{~nm}$ and the region above $750 \mathrm{~nm}$ ). Greater anthocyanin production in petal lobes and the tips of sepals and subtending bracts at cool temperatures explains the reduced reflectance and noticeable color darkening in the visible region (Stiles et al. 2007). Whereas floral reflectance strongly responds to temperature change, leaf reflectance shows little response (fig. A1).

Individual plants partially thermoregulate reproduction by producing poorly reflective (and darker) spikes/flowers in the spring and fall, when it is cool, and highly reflective (and lighter) spikes/ flowers in the summer, when it is warm. Reducing floral and fruit reflectance at cool temperatures increases solar radiative absorption, which warms flowers and developing fruits (Lacey and Herr 2005). Increasing reflectance helps cool flowers and fruits produced at warm temperatures.

Floral-reflectance plasticity is genetically variable within and among populations and is largely explained by variation in the ability to reduce reflectance at cool temperatures (Lacey and Herr 2005; Lacey et al. 2010). Most genotypes produce highly reflective flowers/spikes at warm temperatures. "Strong thermoregulators" reduce reflectance substantially at cool temperatures, while thermally insensitive "thermoconformers" show little to no reduction (e.g., fig. 1). Other genotypes lie between these extremes. Our experiments took advantage of this genetic variation to explore the reproductive consequences of being able to partially thermoregulate reproduction.

We tested the hypothesis that thermoregulation is more adaptive in thermally variable environments that are, on average, cool and short and less adaptive in thermally variable environments that are, on average, warm and long. In nature, the intensity of selection acting on phenotypic plasticity can vary across the range of environments experienced by an organism. In such situations, plasticity should be favored in environments where the frequency of intense selection is high but not when the frequency is low (Levins 1968; Gomulkiewicz and Kirkpatrick 1992; Moran 1992). Lacey et al. (2010) extended this argument to thermal acclimation and thermoregulation in ectotherms by proposing that the selection intensity favoring acclimation/thermoregulation should be high at temperatures where an alteration of internal body temperature could potentially produce a large fitness benefit, for example, at external temperatures that strongly influence metabolic rate. The selection intensity should weaken as the potential fitness benefit of altering body temperature wanes, for example, as the influence of external temperature on metabolic rate declines. Because physiological data indicate that temperature controls metabolic pro-

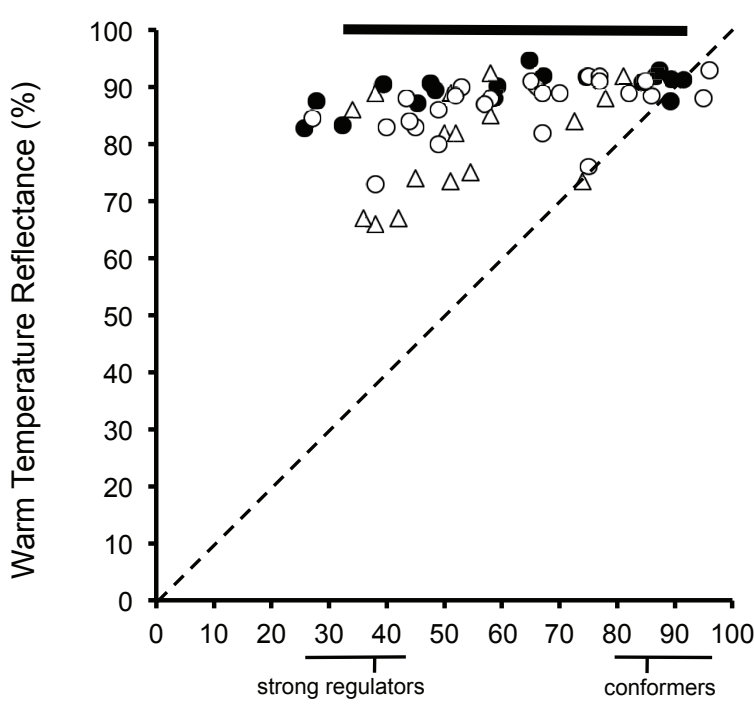

Cool Temperature Reflectance (\%)

Figure 1: Mean percent reflectance at $850 \mathrm{~nm}$ for spikes produced at warm $\left(27^{\circ} \mathrm{C}\right.$ day $/ 20^{\circ} \mathrm{C}$ night $)$ and $\operatorname{cool}\left(15^{\circ} \mathrm{C}\right.$ day $/ 10^{\circ} \mathrm{C}$ night $)$ temperatures for (1) second-generation genotypes scanned during the selection process (circles), (2) the second-generation genotypes chosen for our experiments (filled circles), and (3) a sample of genotypes in a local Greensboro population (triangles). The horizontal bar indicates the range of cool-temperature reflectances for firstgeneration genotypes used in selection process. The dashed line indicates the theoretical line of absolute thermoconformity, that is, no temperature-sensitive reflectance plasticity. See "Methods" for further explanation.

cesses more strongly at cool temperatures than at warm temperatures (e.g., for plants: Covey-Crump et al. 2002; Atkin et al. 2005), Lacey et al. (2010) proposed that thermoregulatory and acclimation ability should be more adaptive as the frequency of cool temperatures experienced in a thermally variable environment increases. (Very hot temperatures are not considered here.) This argument would explain why in many ectotherms, thermal-acclimation ability increases toward the poles and at higher altitudes. Results of a large-scale study of reflectance plasticity in P. lanceolata along latitudinal and altitudinal gradients provide empirical support for the explanation and, in addition, suggest that thermoregulation should be more adaptive where thermally variable environments are short, as well as predominantly cool, and that the adaptive advantage should wane as environments lengthen, as well as warm.

We tested this hypothesis by measuring the effects of floral-reflectance plasticity on reproductive success in $P$. lanceolata at a local scale where the reproductive season thermally varies but is, on average, long and warm. Our 
field site was located in Greensboro, North Carolina. In Greensboro, the reproductive season extends from midApril through August, and 30-year mean monthly temperatures, as well as the peak monthly means, exceed the monthly means of previously sampled southern sea-level European populations (fig. A2, available online). The hypothesis makes two predictions in such an environment: (1) that genotypes with strong reflectance plasticity, that is, strong thermoregulation, should show greater reproductive success during the cool portion(s) of the reproductive season and (2) that this fitness advantage should disappear during the longer, warmer portion of the season, such that genotypes with little thermoregulation have equal or greater reproductive success summed over an entire reproductive season.

Second, we explored the relationship between thermoregulation and reproductive phenology. Researchers have suggested that physiological and phenological traits may be correlated with each other and may represent lifehistory trade-offs (Dunham et al. 1989; Huey et al. 2003; Ragland and Kingsolver 2008; Angilletta 2009; Forrest and Miller-Rushing 2010; Lesica and Kittelson 2010; Robinet and Roques 2010). Therefore, we asked whether thermoregulation and reproductive phenology are correlated with each other and, if so, how the correlation might influence the evolution of thermoregulation and the response to climate change. Recent shifts in the timing of reproduction in temperate species have been strongly correlated with atmospheric warming (e.g., Parmesan 2006; Bertin 2008). These shifts, in addition to thermoregulation, could help ectothermic species buffer the effect of climatic warming. If traits are genetically correlated, then their interaction should determine evolutionary responses to atmospheric warming (Lande 1982; Huey and Bennett 1987; O’Neil 1997; Etterson and Shaw 2001). To explore the association between thermoregulation and phenology, we examined the reproductive phenology of genotypes varying in thermoregulation in Greensboro.

Reproductive timing is typically measured in terms of onset and duration of reproduction, which jointly define a reproductive window (e.g., for plants: Rathcke and Lacey 1985; Körner 2003; Badeck et al. 2004; Kudo 2006; Sherry et al. 2007; Bertin 2008). However, phenology also includes reproductive packaging within the window, for example, timing of clutch (e.g., spike) production (e.g., Dunham et al. 1989). Differences in one or some combination of the above traits could contribute to differences in reproductive phenology. Therefore, we examined the timing of spike and seed production within the reproductive season, in addition to reproductive onset and duration.

\section{Methods \\ Experimental Genotype Selection}

We used 18 experimental hermaphroditic genotypes ranging from strongly thermoregulating genotypes (hereafter "strong thermoregulators") to ones lacking this ability, that is, showing negligible floral-reflectance plasticity (hereafter referred to as "thermoconformers"). Genotypes were produced by two generations of selection to augment the number of extremes (fig. 1). Plants $(N=111 ; \geq 0.5 \mathrm{~m}$ apart) were haphazardly collected from seven North Carolina populations (two in Durham, one in Hillsborough, two in Greensboro, and two in Black Mountain/Swannanoa) and then induced to produce flowers at a cool $\left(15^{\circ} \mathrm{C}\right.$ day $/ 10^{\circ} \mathrm{C}$ night $)$ temperature in a growth chamber. We scanned 81 plants to sample the floral color range in each population (sample sizes: 5 and 13 from Durham, 11 from Hillsborough, 17 and 14 from Greensboro, and 16 and 5 from Black Mountain/Swannanoa). One spike per plant was scanned twice by means of a spectrophotometer with an integrating sphere to measure percent reflectance (method in Lacey and Herr 2005). On the basis of mean cool-temperature spike reflectance at $850 \mathrm{~nm}$, we assigned a sample of plants to each of three reflectanceplasticity groups ( $N=13,13$, and 10 for low-, medium-, and high-plasticity groups, respectively). Groups were placed in separate growth chambers for random pollination and seed production at an intermediate temperature (16-h day at $20^{\circ} \mathrm{C}, 8-\mathrm{h}$ night at $\left.15^{\circ} \mathrm{C}\right)$ and otherwise similar environmental conditions (e.g., fertilized with halfstrength Hoagland's solution three times per week). The species is self-incompatible and primarily wind pollinated.

Three to five offspring per maternal parent were induced to flower in a growth chamber at a cool temperature $\left(15^{\circ}\right.$ / $10^{\circ} \mathrm{C}$ ). Of these, we chose 44 that spanned the range of floral color variation and scanned one spike per chosen plant. Then we raised the temperature $\left(27^{\circ} / 20^{\circ} \mathrm{C}\right)$, allowed the selected genotypes to produce spikes at the warm temperature, and again scanned one spike per plant (fig. 1; circles represent offspring scanned at both temperatures). Floral-reflectance plasticity for each genotype was calculated as the absolute difference (magnitude of change) in percent reflectance between the spikes produced at the two temperatures. Final experimental genotypes (fig. 1, filled circles) were chosen from these scanned offspring on the basis of floral reflectance at $850 \mathrm{~nm}$. Experimental genotypes included progeny of maternal parents originating from six of the seven natural populations. Each end of the plasticity continuum included at least one descendent of a parent collected from the highest- and lowest-altitude North Carolina populations that we sampled (Black Mountain and Durham: 731 and $134 \mathrm{~m}$ altitude, respectively). Experimental genotypes extended the range of plas- 
ticities measured for a natural population that we had previously sampled in Greensboro (fig. 1, triangles), and they extended the range observed in first-generation plants used for selection (fig. 1, horizontal bar).

Experimental clones (cuttings) were made from stock genotypes maintained in a greenhouse. The general procedure was to grow clones at $20^{\circ} / 15^{\circ} \mathrm{C}(10$-h day, 14 -h night) for 6-8 weeks to promote vegetative growth. If necessary, we then increased the day length to $16 \mathrm{~h}$ to induce flowering. Experimental clones were similar in size (E. P. Lacey, personal observation).

We conducted two experiments in a field where Plantago lanceolata grows naturally in Greensboro. Mean monthly temperatures during the reproductive season in Greensboro slightly exceed those of the warmest sites previously sampled in southern Europe (fig. A2). In addition, genotypes were used for a growth-chamber experiment to help interpret the results of one of the field experiments.

\section{Common-Garden Experiment}

In March 2004 and February 2005, we randomly transplanted four clones per genotype from growth chambers to field plots and measured the natural timing of flower and seed production in 2004 and 2005. We collected data from three cohorts, which we identify by transplant year and flowering year: 04-04 for clones transplanted in 2004 and flowering in 2004, 04-05 for clones transplanted in 2004 and flowering in 2005, and 05-05 for clones transplanted in 2005 and flowering in 2005. Field plots were located in a regularly mowed field that contained grasses and weedy forbs, including $P$. lanceolata. For each clone each week, we marked all new spikes. Spikes were collected when mature, assessed for grasshopper damage and fungal infection, and then weighed. Regression equations (fig. 4; Lacey et al. 2003) relating seed production to spike mass for undamaged, grasshopper-damaged, and fungusinfected spikes were used to estimate seed production per spike. Estimated seed numbers for all spikes produced per clone per week were then summed. One genotype did not flower in 2004, and another was infected by fungus in 2005. Consequently, sample sizes for the 04-04, 04-05, and $05-05$ cohorts were 17,18 , and 17 genotypes, respectively.

\section{Phenotypic-Manipulation Experiment}

At three times in 2008, we used growth chambers to photoperiodically induce (16-h days) two or three clones per genotype to produce spikes with "cool-induced" $\left(15^{\circ} /\right.$ $\left.10^{\circ} \mathrm{C}\right)$ and "warm-induced" $\left(27^{\circ} / 20^{\circ} \mathrm{C}\right)$ floral phenotypes (fig. 1). All plants received half-strength Hoagland's solution once per day. After flowering had begun, we marked
1-3 spikes with flower buds but no emerging stigmas on each clone, recorded spike lengths (mean $[\mathrm{SD}]=1.40$ [0.23] cm; range: $0.8-2.1 \mathrm{~cm}$ ), and randomly transplanted clones into freshly plowed field plots (adjacent to the common-garden plots) to allow for natural pollination and seed set. We collected mature spikes and counted their seeds.

Clones were transplanted in mid-April, late May, and early July (1-3 clones/genotype/induction temperature/ transplant time). Field plants were watered when necessary to prevent desiccation. Temperatures during the time of the experiment resembled 30-year averages, except for an unusual hot spell in early June (fig. A3, available online). Unfortunately, a malfunctioning watering system caused the May transplants to suffer a week of drought stress during that hot spell.

Of the 216 clones placed in the field, data were collected from 207 (479 spikes total). Some clones were lost because their spikes did not survive handling and transplanting. Also, a few spikes on usable clones were lost for the same reason and because of fungal infection and herbivory, most likely from a mammal. Lost spikes were scattered across experimental genotypes and transplant times. Grasshoppers, which in some years can heavily damage developing fruits, were negligible in this year, likely because of a severe drought in the southeastern United States in 2006-2007, which may have caused a drastic reduction in the local grasshopper population. Grasshoppers did not damage the experimental spikes.

\section{Growth-Chamber Experiment}

Data from the phenotypic-manipulation experiment suggested that reflectance plasticity might affect seed production per spike at cool temperatures indirectly by influencing the initial number of flowers produced on a spike (i.e., potential clutch size). Therefore, in 2010 we conducted a growth-chamber experiment to test whether spike size is associated with reflectance plasticity at cool temperatures. Spike length at the time when flowering begins on a spike is a good indicator of potential clutch size (E. P. Lacey, unpublished data). Three to five clones per genotype were randomly placed in a cool-temperature $\left(15^{\circ} /\right.$ $\left.10^{\circ} \mathrm{C}\right)$ chamber and induced to flower $(N=64)$. Plants received half-strength Hoagland's solution once per day and were checked three times per week for flower initiation. At initiation (appearance of a stigma on a spike), spike length and width were measured to the nearest 0.5 $\mathrm{mm}$. We counted the number of leaves longer than $2 \mathrm{~cm}$ and measured the longest leaf length and width. Leaf data were used to estimate total leaf area, our proxy for plant biomass (equation in Lacey 1996). Subsequently, we mea- 
sured the length and width of five additional spikes (the first six per plant).

\section{Statistical Analysis}

All statistical analyses were performed in SAS, version 9.1 (PROC MIXED). In the common-garden experiment, interactions between transplant and flowering years (fixed factors) were statistically significant for all dependent variables. Therefore, we analyzed the phenological data for each cohort separately. We used linear mixed models to measure the effects of reflectance plasticity (of each genotype) on flowering onset (week of first spike appearance), flowering duration (number of weeks new spikes were produced), and the temporal pattern of spike production within the reproductive season (median week of spike production). In a second analysis, we used linear mixed models to measure the effects of week (weeks 20, $23,28,35$, or 38 ) and reflectance plasticity on log-transformed cumulative spike and seed production. Dependent variables were analyzed separately.

For the phenotypic-manipulation experiment, linear mixed models were used to examine seed production per spike as a function of the reflectance plasticity (i.e., degree of thermoregulation) of a genotype, induction temperature, and transplant time. Log transformation of seeds per spike was unnecessary. The analysis was run twice, with and without mean initial spike length per clone as a covariate. Pairwise means comparisons were used to determine whether the effect of thermoregulation on seed production changed over the reproductive season. For the growth-chamber experiment, we used a least squares regression model to assess the effect of reflectance plasticity on spike length and width. Developmental order of each spike and total leaf area per plant were included as covariates.

For the phenotypic-selection analyses of the commongarden data, we used quadratic regression models to examine the effect of plasticity on two measures of reproductive fitness per cohort: cumulative spike and cumulative seed production at week 38 , by which time spike production had ceased for all cohorts. Also, we used quadratic regression models for the phenotypic-selection analysis of the phenotypic-manipulation data to examine the effect of plasticity on seed production per spike. We ran the analyses for spike production and seeds per spike because both contribute to cumulative seed production. In a life-history context, reproductive success is determined by clutch (spike) number and clutch size (seeds per spike). We wished to examine the relative contributions of each to seasonal reproductive success.

Analyses were performed separately on each fitness proxy. Plasticity was standardized $($ mean $=0$, variance $=$
1), and untransformed relative fitness was calculated by dividing cumulative spike or seed production at week 38 by mean cumulative production per transplant by flowering-year combination (1-2). The standardized linear $(\beta)$ and quadratic $(\gamma)$ selection gradients were estimated, and the parameter estimate for $\gamma$ was doubled (see Stinchcombe et al. 2008). Because the residuals of the regression analyses suggested that errors were not normally distributed, we examined the $P$ values from separate analyses of untransformed and log-transformed relative fitness (Lande and Arnold 1983; Mitchell-Olds and Shaw 1987). The $P$ values were very similar and the conclusions the same; therefore, we report the $P$ values for untransformed data. A statistically significant linear selection gradient $(\beta)$ indicates evidence of directional selection. A statistically significant quadratic gradient $(\gamma)$ indicates evidence of curvature in the fitness function. If the optimal fitness is associated with an intermediate trait value (e.g., intermediate reflectance plasticity), then there is evidence of stabilizing selection.

\section{Results \\ Common-Garden Experiment}

In 2004, spike/flower production spanned Julian weeks 22 38 (May-September). Ten genotypes were flowering by week 23, and all were flowering by week 25 (fig. 2). In 2005, spike/flower production for March 2004 transplants spanned weeks 17-35. By weeks 19 and 21, 12 and all genotypes were flowering, respectively. Spike/flower production for February 2005 transplants spanned weeks 1738. By week 17, 14 of 17 genotypes were flowering. Onset and duration of spike production were not associated with reflectance plasticity in any cohort (table 1).

In contrast, plasticity did influence the timing of spike production within the reproductive window (table 1). The effect changed, however, over the reproductive season, and the seasonal pattern differed among cohorts (fig. 2). In general, during the early reproductive weeks, highly plastic genotypes produced more spikes. This pattern is observed in the 04-04 and 04-05 cohorts, where $\log$ (cumulative spike production) was statistically and positively associated with plasticity (04-04 cohort: slope(wk 23) $=0.0128$ [fig. $2 A], P=.0424 ; 04-05$ cohort: slope(wk 19) $=0.0140$, $P=.0065$, slope(wk 20) $=0.0190$ [fig. $2 B$ ], $P=.0004$, slope(wk 21) $=0.0150, P=.0039)$. This relationship reversed itself in subsequent weeks, as positive slopes became negative. For the 05-05 cohort, there was moderate evidence of negative slopes in the last weeks of summer (slope $($ wk 33) $=-0.0104, P=.0644$; slope $($ wk 36) $=$ $-0.0109, P=.0520$; slope(wk 38) $=-0.0110$ [fig. 2C], $P=.0502)$. Consistent with the above analyses, median 
A) ‘04 in '04
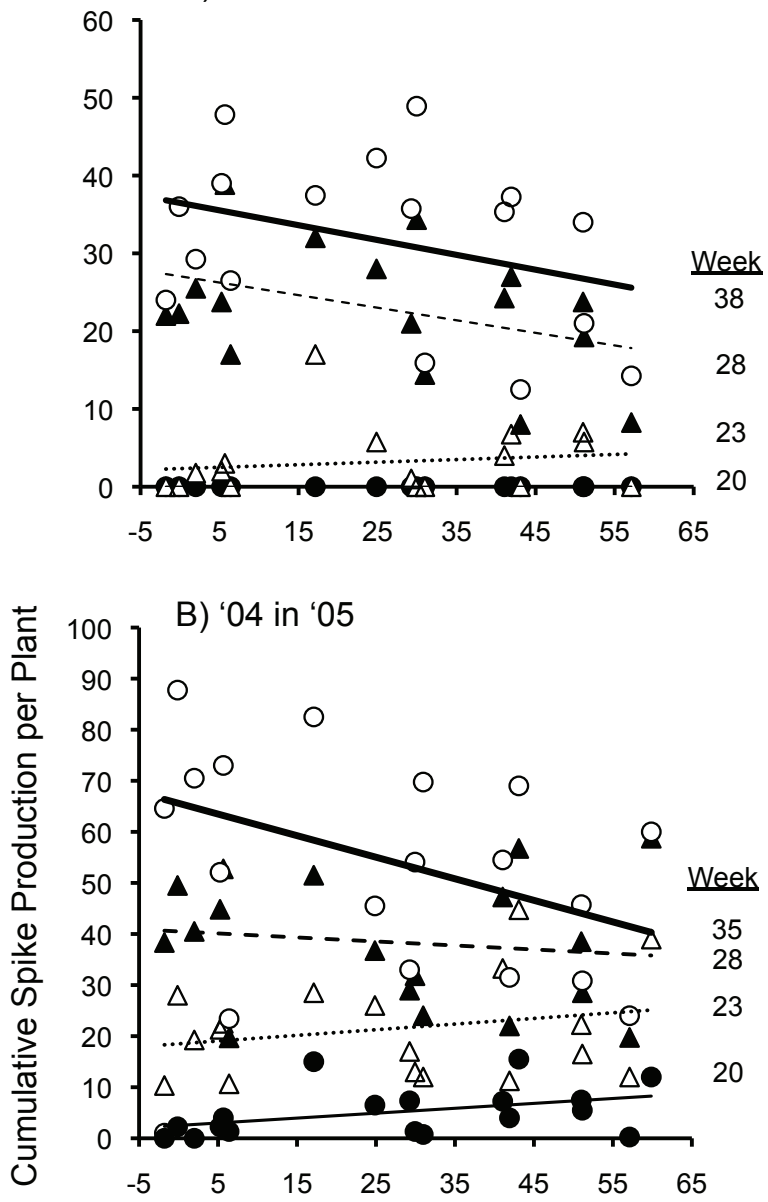

C) ‘ 05 in '05

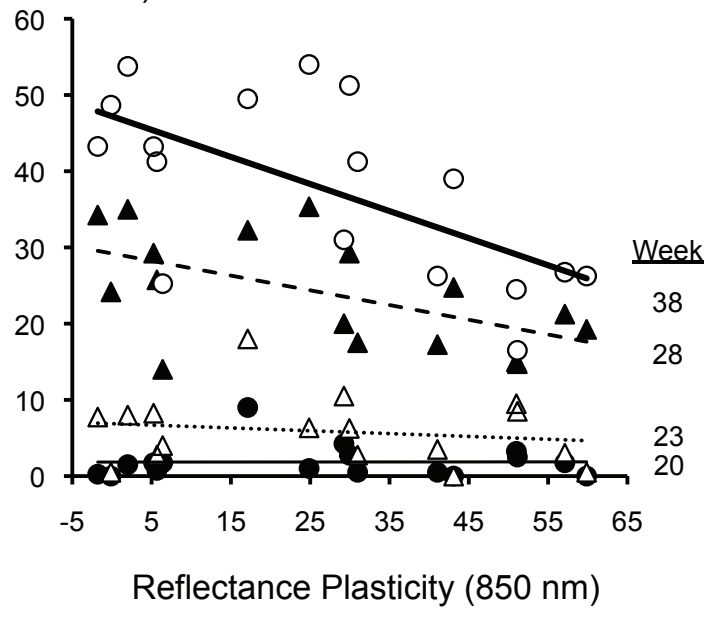

Figure 2: Linear relationships between reflectance plasticity and mean cumulative spike production per clone (3-4 clones/genotype/ wk) at weeks 20 (filled circles, thin solid line), 23 (open triangles, dotted line), 28 (filled triangles, dashed line), and 35 or 38 (open week of spike production was later in low-plasticity genotypes in all cohorts and significantly later in two cohorts (table 1).

The timing of seed production paralleled the pattern for spikes in the 04-04 and 04-05 cohorts (fig. 3). Linear mixed-model analyses showed that early in the season, highly plastic genotypes produced more seeds. Statistical evidence that cumulative seed production was positively associated with thermoregulation was moderate to strong (04-04 cohort: slope $($ wk 23) $=0.0334$ [fig. 3A], $P=$ .0008 ; 04-05 cohort: slope(wk 19) $=0.0501, P<.0001$, slope $(w k$ 20) $=0.0541$ [fig. 3B], $P<.0001$, slope $(w k$ $21)=0.0186, P=.0587)$. As with spikes, this positive relationship became negative, although the negative slopes were never statistically different from 0 .

Phenotypic-selection analyses of seasonal reproductive output showed that the pattern of selection depended on the fitness proxy. When total spike production was used as the proxy, direct linear selection $(\beta)$ favored the absence of plasticity, that is, thermoconformity (fig. 2). There was strong statistical evidence for $04-05$ and $05-05$ cohorts and moderate evidence for the 04-04 cohort that linear selection gradients were negative (table 2). There was no evidence of quadratic selection gradients on spike production.

When total seed production was used as the fitness proxy, evidence of linear selection favoring no plasticity was moderate and was observed only for the 04-04 cohort (fig. $3 A$; table 2). In contrast, there was strong statistical evidence of nonlinear selection $(\gamma)$ for the 05-05 cohort (fig. 3C; table 2). The negative gradient and inspection of the data indicated stabilizing selection favoring intermediate plasticity, with a slight skewing of the optimum favoring no plasticity (optimal plasticity value $=24.2$ ). The 04-04 cohort showed a similar, although nonsignificant, curvilinear pattern $(P=.1647)$.

\section{Phenotypic-Manipulation Experiment}

Linear mixed-model analyses did not detect any effect of plasticity on per-spike seed number in warm-induced spikes for any transplant group (fig. 4A). This was expected because all genotypes produce highly reflective flowers/ spikes at warm temperatures. Warm-induced (highly reflective) spikes on April and July transplants produced similar per-spike seed numbers and more seeds than did

circles, thick solid line) for the 04-04 (A), 04-05 (B), and 05-05 (C) cohorts. Respective slopes for weeks $20,23,28$, and 35 or 38 for the 04-04 cohort: $0.0003,0.0334,-0.0073$, and -0.0049 ; for the $04-05$ cohort: $0.0541,0.0063,-0.0011$, and -0.0036 ; for the $05-05$ cohort: $-0.0134,-0.0276,-0.0018$, and -0.004 . 
Table 1: Effects of reflectance plasticity on phenological traits in Plantago lanceolata

\begin{tabular}{lcll}
\hline $\begin{array}{l}\text { Transplant year } \times \\
\text { flowering year }\end{array}$ & Onset & Duration & Median week \\
\hline $2004 \times 2004$ & -.0134 & -.0068 & -.0028 \\
$2004 \times 2005$ & -.0140 & -.0257 & $-.0439^{* *}$ \\
$2005 \times 2005$ & -.0034 & -.01535 & $-.0324^{* *}$ \\
\hline
\end{tabular}

Note: Onset $=$ onset of spike/flower production in weeks; duration $=$ reproduction duration in weeks; median week $=$ median week of spike production. Values are slopes of regression lines, shown by cohort (transplant year $\times$ flowering year combination). $P>.10$ for all slope estimates except as noted.

${ }^{* *} P<.01$.

May transplants, which had suffered a temporary drought during seed development (fig. 4A).

Linear mixed-model analyses of cool-induced spikes showed a different pattern. Plasticity did not affect perspike seed number for May and July transplants (fig. 4B). However, there was evidence of increased seed production associated with increasing reflectance plasticity for April transplants (fig. $4 B ; P=.023$ ). The coolest part of the reproductive season occurs in April (fig. A3). Slope comparisons showed a statistically significant difference in slope between April and July (April slope = 1.141; July slope $=-0.160$; estimated difference in slopes $=1.300$, $P=.045)$. Statistical evidence of a difference between April and July slopes was moderately convincing even after differences in initial spike length were accounted for in the regression model (estimated difference $=1.252$, $P=.051)$. The slope of May transplants was intermediate between the April and July slopes, and the difference between the April and May slopes (1.06) was moderate $(P=.087)$.

Phenotypic-selection analyses of per-spike seed number showed evidence of strong directional selection favoring high plasticity among cool-induced phenotypes transplanted in April but strong stabilizing selection favoring intermediate plasticity among cool-induced phenotypes transplanted in July (fig. 4; table 3). Thus, over the course of the reproductive season, the optimal plasticity shifted toward less plasticity. In midsummer, per-spike seed production was noticeably lower for high-plasticity genotypes than for intermediate-plasticity genotypes. Also, selection favored intermediate plasticity for warm-induced phenotypes transplanted in May.

\section{Growth-Chamber Experiment}

The length of cool-induced spikes at the onset of flowering, that is, potential clutch size, was positively associated with plasticity (slope $=0.0084, P=.0173$ ), independent of the order in which spikes were produced $(P=.0365)$. Effects
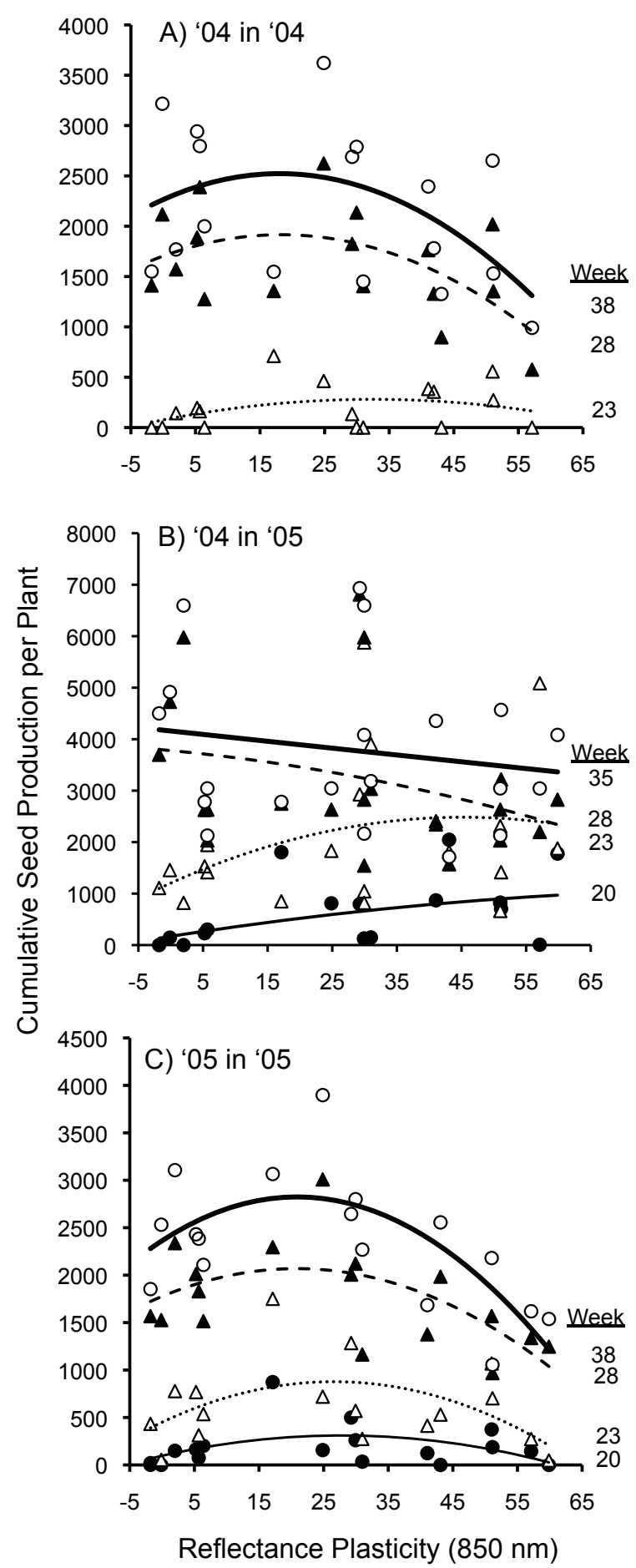

Figure 3: Second-order polynomial relationships between reflectance plasticity and mean cumulative seed production per clone (3-4 clones/genotype/wk) at weeks 20 (filled circles, thin solid line), 23 (open triangles, dotted line), 28 (filled triangles, dashed line), and 35 or 38 (open circles, thick solid line) for the 04-04 $(A), 04-05(B)$, and $05-05(C)$ cohorts. 
Table 2: Selection coefficients for reflectance plasticity in Plantago lanceolata, using total seasonal spike and seed production at week 38 as fitness proxies

\begin{tabular}{cllllc}
\hline & \multicolumn{2}{c}{ Spikes } & & \multicolumn{2}{c}{ Seeds } \\
\cline { 2 - 3 } Transplant year $\times$ flowering year & Linear $(\beta)$ & Quadratic $(2 \gamma)$ & & Linear $(\beta)$ & Quadratic $(2 \gamma)$ \\
\hline $2004 \times 2004$ & $-.1198^{\mathrm{a}}$ & -.2406 & & $-.1188^{\mathrm{b}}$ & -.2558 \\
$2004 \times 2005$ & $-.1738^{* *}$ & -.0018 & & -.0491 & -.2066 \\
$2005 \times 2005$ & $-.2025^{* * *}$ & -.1778 & & -.0537 & $-.4596^{* *}$ \\
\hline
\end{tabular}

Note: $P$ values are shown for moderately and strongly significant effects.

${ }^{a} P=.070$.

b $P=.091$

${ }^{* *} P<.01$.

${ }^{* * *} P<.0001$.

of leaf area and interactions between plasticity and order were nonsignificant, as were all effects related to width.

\section{Discussion}

Few manipulative field experiments have been conducted to explore the adaptive advantage of thermal acclimation and thermoregulation in nature. Kingsolver and Huey artificially manipulated wing color (i.e., the thermoregulatory mechanism) in Pontia occidentalis butterflies and re- leased them into the field in spring and summer (Kingsolver 1995; Kingsolver and Huey 1998). Loeschcke and Hoffmann (2007) and Kristensen et al. (2008) released cold- and heat-treated Drosophila melanogaster into the field at different ambient temperatures. These experiments have provided evidence that thermal acclimation and thermoregulation affect survival and food-finding ability. Our field experiments add to this work by using selected genotypes to examine the effects of thermoregulation on reproduction.

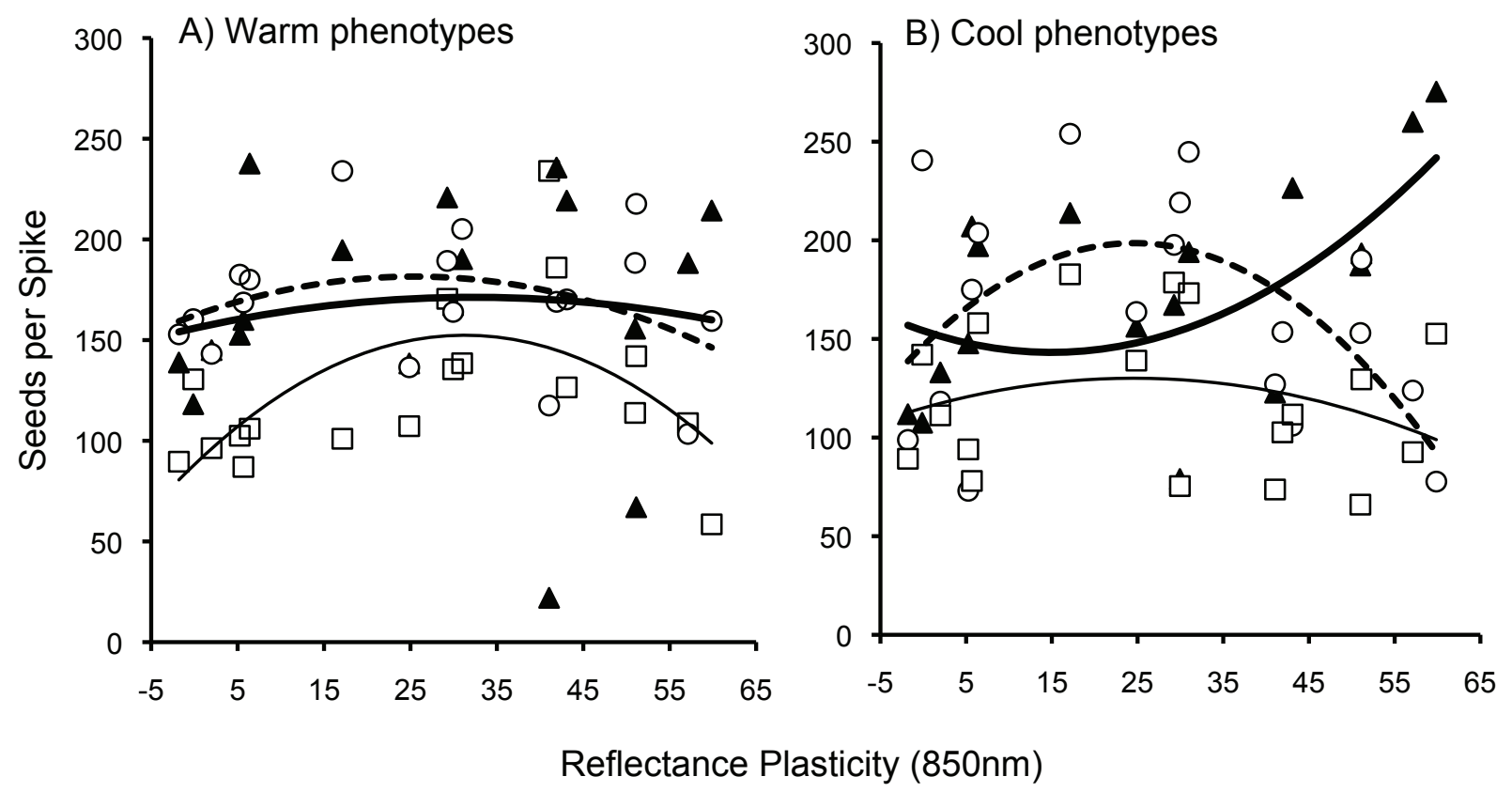

Figure 4: Second-order polynomial relationships between reflectance plasticity and mean seed production per spike on clones of genotypes induced to produce warm-temperature $(A)$ and cool-temperature $(B)$ phenotypes. $A$, Estimated linear slopes (with $P$ values) for April (triangles, thick solid line), May (squares, thin solid line), and July (circles, dashed line) transplants are 0.5074 (.298), 0.6506 (.175), and -0.0875 (.858), respectively. $B$, Slope values $(P)$ for April, May, and July transplants are $1.1406(.023),-0.058(.907)$, and $-0.1599(.759)$, respectively. 
Table 3: Selection coefficients for reflectance plasticity in Plantago lanceolata, using seeds per spike as the fitness proxy

\begin{tabular}{lcc}
\hline & \multicolumn{2}{c}{ Seeds per spike } \\
\cline { 2 - 3 } $\begin{array}{l}\text { Induction temperature } \times \\
\text { transplant time }\end{array}$ & $\begin{array}{c}\text { Linear } \\
(\beta)\end{array}$ & $\begin{array}{c}\text { Quadratic } \\
(2 \gamma)\end{array}$ \\
\hline Cool $\times$ April & $.1396^{\mathrm{a}}$ & -.1788 \\
Cool $\times$ May & -.0338 & -.1244 \\
Cool $\times$ July & -.0922 & $-.4448^{* *}$ \\
Warm $\times$ April & .0909 & -.1126 \\
Warm $\times$ May & .0505 & $-.3452^{*}$ \\
Warm $\times$ July & -.0053 & -.1972 \\
\hline
\end{tabular}

Note: $P$ values are shown for moderately and strongly significant effects.

${ }^{a} P=.052$.

${ }^{*} P<.05$.

${ }^{* *} P<.01$.

Our data support the hypothesis that strong thermoregulation is adaptive in ectotherms in predominantly short, cool, thermally variable environments but becomes less adaptive as thermally variable environments lengthen and warm. Consistent with one of the hypothesis's predictions, strong thermoregulators did produce more spikes, more seeds per spike, and more seeds per plant during the spring, when ambient temperatures were cool. Directional selection favored strong thermoregulators in terms of per-spike seed production. The growth-chamber experiment showed that at a cool temperature, thermoregulators produce longer spikes, indicative of more flowers per spike at the initiation of flowering, and the phenotypic-manipulation experiment showed that the strong effect of reflectance plasticity on per-spike seed production persisted even after initial spike length was accounted for. Thus, both initial flower number per spike and continued seed production appear to contribute to the greater perspike seed production in strong thermoregulators.

Consistent with the second prediction, the reproductive advantage of strong thermoregulators disappeared as the season progressed. Thermoconformers produced more spikes than did strong thermoregulators in the latter part of the season, and this offset the initial reproductive advantage of strong thermoregulators. Directional selection strongly favored thermoconformers when fitness was measured in terms of seasonal spike production. Phenotypicselection analyses of total seasonal seed production, however, showed little evidence of a selective advantage for either thermoconformers or strong thermoregulators. Instead, in two of three cohorts, there was evidence of stabilizing selection favoring intermediate thermoregulation. Intermediate thermoregulators may have benefited because they had a mildly greater ability than did thermoconformers to thermoregulate in the spring and because they produced a few more spikes than did thermoregulators in midsummer. The multiplicative effect of increased spike number and per-spike seed number might have given intermediate thermoregulators a slight advantage. This scenario would explain why the range of plasticities observed in a previously studied Greensboro population (Lacey and Herr 2005) was centered about an intermediate plasticity value and was narrower than the range observed after two generations of selection to produce the genotypes used in our experiments (fig. 1).

Cool-temperature reflectance alone largely explains the variation in floral-reflectance plasticity, that is, thermoregulation, in this species (fig. 1). Therefore, one might ask whether selection is acting on plasticity or on cooltemperature reflectance alone. Results of the phenotypicselection analyses support the former scenario. First, for cool-induced phenotypes produced in the phenotypicmanipulation experiment, the directional selection that favored strong thermoregulators in April shifted to stabilizing selection favoring intermediate thermoregulators in July (fig. 4B). The benefit of producing weakly reflective flowers in April became a liability in July; the cost of producing more-reflective flowers in April became an asset in July. Second, there was no detectable effect of thermoregulation, that is, reflectance plasticity, on per-spike seed production among warm-induced flowers/spikes for July transplants (fig. 4A). When strong thermoregulators were artificially induced to produce highly reflective flowers, which they normally produce at warm temperatures in July, the fitness difference between strong and intermediate thermoregulators observed in cool-induced phenotypes disappeared. These combined results indicate that natural selection is functioning at both warm and cool temperatures and that plasticity is the target of selection.

When viewed over the whole reproductive season, our field environment favored some plasticity rather than thermoconformity in two of three cohorts (fig. 2). However, we used only thermoconformers with highly reflective flowers. We found only three thermoconformers constitutively producing weakly reflective flowers in several hundred European genotypes sampled (Lacey et al. 2010), and we found none when selecting genotypes for this study. Warm-temperature reflectance was not measured in the parental generation or for the 44 progeny selected for scanning. Thus, there was no known bias against weakly reflective thermoconformers. Yet among the scanned progeny we found only one thermoconformer that produced flowers having less than $80 \%$ reflectance at both warm and cool temperatures (fig. 1, open circle along dashed line). This genotype resembled the least-reflecting thermoconformer in a sample of genotypes from a natural Greensboro population (fig. 1, triangle along dashed line). There 
appears to be strong selection against genotypes that constitutively produce weakly reflective flowers.

Thus far, two lines of evidence support the view that thermoregulation in Plantago lanceolata is heritable. First, previous studies show that thermoregulation is genetically variable within and among P. lanceolata populations (Lacey and Herr 2005; Lacey et al. 2010). Second, with one generation of selection to produce our experimental genotypes, we were able to expand the range of cool-temperature reflectances (fig. 1). These observations suggest that additional experiments to clarify the genetic basis for reflectance plasticity would be valuable.

Our experimental results showed a negative phenotypic correlation between thermoregulation and phenology. Several mechanisms could explain this association. Early in the reproductive season, the warmer internal flower/fruit temperatures in strong thermoregulators might accelerate the rate of flower and fruit development. Consequently, stronger thermoregulators may exhaust their resources more quickly. Alternatively or additionally, thermoregulation might be energetically costly. Increased anthocyanin content explains floral darkening at cool temperatures in P. lanceolata (Stiles et al. 2007), and higher anthocyanin production might reduce resources available for offspring production late in the reproductive season and over the whole season. Pleiotropically acting genes may determine both thermal plasticity in floral reflectance and flowering phenology, possibly in one of the manners described above. Alternatively, selection may favor earlier flowering in strong thermoregulators. Previous studies showed evidence of opposing multigenerational selection pressures acting on reproductive timing in P. lanceolata in the Piedmont of North Carolina (Lacey et al. 2003). Warmer temperatures experienced in mid- to late summer substantially improve offspring fitness, which favors delayed reproduction (Lacey and Herr 2000). However, higher seed predation during the latter part of the reproductive season favors accelerated reproduction (Lacey et al. 2003). These opposing pressures should be comparable for all individuals, except that thermoregulating individuals can warm gametes and developing offspring, thereby partially offsetting the negative effects of early-season cool temperatures on offspring fitness (Lacey et al. 2003). Consequently, selection might drive strongly thermoregulating genotypes to reproduce earlier than genotypes lacking that ability.

Studies of reproductive phenology, including climatechange studies, have typically focused on reproductive onset and duration (e.g., Parmesan 2006; Sherry et al. 2007; Bertin 2008; Todd et al. 2011). Our data showed no association between thermoregulation and either phenological trait. This is not surprising because, as for many other organisms (Bradshaw and Holzapfel 2001; Körner and Basler 2010), reproduction in P. lanceolata is largely con- trolled by photoperiod. However, strong thermoregulators produced more and larger spikes (clutches) earlier in the reproductive window than did thermoconformers. These data suggest that photoperiodically controlled species may have phenological flexibility to respond to climate change by shifting the timing of clutch production and clutch size within a reproductive season. Future phenological studies might consider such responses.

Species' responses to atmospheric warming are likely to be complex because distinctions between phenotypic plasticity and local adaptation are often unclear (Gienapp et al. 2008). Our study suggests that it is important to consider the range of induced phenotypes in a plastic individual relative to the fixed phenotype of a nonplastic individual in a population, in addition to any cost of plasticity. For example, a thermoregulator that cannot lower internal body temperature at warm ambient temperatures, regardless of its ability to raise body temperature at cool ambient temperatures, as in P. lanceolata, should suffer reduced fitness relative to a thermoconformer in a warming world, assuming some cost of thermoregulation. Genetically variable populations should evolve toward thermoconformity. If a negative phenotypic correlation between thermoregulation and reproductive phenology, as observed in P. lanceolata, also reflects a genetic correlation, then the correlation should accelerate the shift toward thermoconformity. These implications reinforce the need to better characterize thermally sensitive traits and their interactions.

\section{Acknowledgments}

We thank J. Kingsolver and two anonymous reviewers for their helpful comments on a previous draft of this article; the many undergraduates, particularly K. Clippard, K. Dukes, and E. Fender, who helped with plant maintenance and data collection and entry; and the National Science Foundation for financial support (DEB-0236526 to E.P.L.).

\section{Literature Cited}

Angilletta, M. J., Jr. 2009. Thermal adaptation: a theoretical and empirical synthesis. Oxford University Press, New York.

Angilletta, M. J., Jr., A. F. Bennett, H. Guderley, C. A. Navas, F. Seebacher, and R. S. Wilson. 2006. Coadaptation: a unifying principle in evolutionary thermal biology. Physiological and Biochemical Zoology 79:282-294.

Atkin, O. K., D. Bruhn, V. M. Hurry, and M. G. Tjoelker. 2005. The hot and the cold: unravelling the variable response of plant respiration to temperature. Functional Plant Biology 32:87-105.

Badeck, F.-W., A. Bondeau, K. Böttcher, D. Doktor, W. Lucht, J. Schaber, and S. Sitch. 2004. Responses of spring phenology to climate change. New Phytologist 162:295-309. 
Bertin, R. I. 2008. Plant phenology and distribution in relation to recent climate change. Journal of the Torrey Botanical Society 135 : 126-146.

Bradshaw, W. E., and C. M. Holzapfel. 2001. Genetic shift in photoperiodic response correlated with global warming. Proceedings of the National Academy of Sciences of the USA 98:14509-14511.

Chevin, L., R. Lande, and G. M. Mace. 2010. Adaptation, plasticity, and extinction in a changing environment: towards a predictive theory. PloS Biology 8:e1000357.

Chown, S. L., A. A. Hoffmann, T. N. Kristensen, M. J. Angilletta Jr., N. C. Stenseth, and C. Pertoldi. 2010. Adapting to climate change: a perspective from evolutionary physiology. Climate Research 43: 3-15.

Covey-Crump, E. M., R. G. Attwood, and O. K. Atkin. 2002. Regulation of root respiration in two species of Plantago that differ in relative growth rate: the effect of short- and long-term changes in temperature. Plant, Cell and Environment 25:1501-1513.

Deutsch, C. A., J. J. Tewksbury, R. B. Huey, K. S. Sheldon, C. K. Ghalambor, D. C. Haak, and P. R. Martin. 2008. Impacts of climate warming on terrestrial ectotherms across latitude. Proceedings of the National Academy of Sciences of the USA 105:6668-6672.

Dunham, A. E., B. W. Grant, and K. L. Overall. 1989. Interfaces between biophysical and physiological ecology and the population ecology of the terrestrial vertebrate ectotherms. Physiological Zoology 62:335-355.

Etterson, J. R., and R. G. Shaw. 2001. Constraint to adaptive evolution in response to global warming. Science 294:151-154.

Forrest, J., and A. J. Miller-Rushing. 2010. Toward a synthetic understanding of the role of phenology in ecology and evolution. Philosophical Transactions of the Royal Society B: Biological Sciences 365:3101-3112.

Gienapp, P., C. Teplitsky, J. S. Alho, J. A. Mills, and J. Merilä. 2008. Climate change and evolution: disentangling environmental and genetic responses. Molecular Ecology 17:167-178.

Gomulkiewicz, R., and M. Kirkpatrick. 1992. Quantitative genetics and the evolution of reaction norms. Evolution 46:390-411.

Gunderson, C. A., K. H. O'Hara, C. M. Campion, A. V. Walker, and N. T. Edwards. 2010. Thermal plasticity of photosynthesis: the role of acclimation in forest responses to a warming climate. Global Change Biology 16:2272-2286.

Helmuth, B., J. G. Kingsolver, and E. Carrington. 2005. Biophysics, physiological ecology, and climate change: does mechanism matter? Annual Review of Physiology 67:177-201.

Hofmann, G. E., and A. E. Todgham. 2010. Living in the now: physiological mechanisms to tolerate a rapidly changing environment. Annual Review of Physiology 72:127-145.

Huey, R. B., and A. F. Bennett. 1987. Phylogenetic studies of coadaptation: preferred temperatures versus optimal performance temperature of lizards. Evolution 41:1098-1115.

Huey, R. B., P. E. Hertz, and B. Sinervo. 2003. Behavioral drive versus behavioral inertia in evolution: a null model approach. American Naturalist 161:357-366.

IPCC (Intergovernmental Panel on Climate Change). 2007. Climate Change 2001: The Scientific Basis. United Nations Environmental Programme.

Kearney, M., and W. Porter. 2009. Mechanistic niche modeling: combining physiological and spatial data to predict species' ranges. Ecology Letters 12:334-350.

Kearney, M., R. Shine, and W. P. Porter. 2009. The potential for behavioral thermoregulation to buffer "cold-blooded" animals against climate warming. Proceedings of the National Academy of Sciences of the USA 106:3835-3840.

Kingsolver, J. G. 1995. Fitness consequences of seasonal polyphenism in western white butterflies. Evolution 49:942-954.

Kingsolver, J. G., and R. B. Huey. 1998. Evolutionary analyses of morphological and physiological plasticity in thermally variable environments. American Zoologist 38:545-560.

Knies, J. L., J. G. Kingsolver, and C. L. Burch. 2009. Hotter is better and broader: thermal sensitivity of fitness in a population of bacteriophages. American Naturalist 173:419-430.

Körner, C. 2003. Alpine plant life: functional plant ecology of high mountain ecosystems. Springer, Berlin.

Körner, C., and D. Basler. 2010. Phenology under global warming. Science 327:1461-1462.

Kristensen, T. N., A. A. Hoffmann, J. Overgaard, J. G. Sorensen, R. Hallas, and V. Loeschcke. 2008. Costs and benefits of cold acclimation in field released Drosophila. Proceedings of the National Academy of Sciences of the USA 105:216-221.

Kudo, G. 2006. Flowering phenologies of animal-pollinated plants: reproductive strategies and agents of selection. Pages 139-158 in L. D. Harder and S. C. H. Barrett, eds. The ecology and evolution of flowers. Oxford University Press, Oxford.

Lacey, E. P. 1996. Parental effects in Plantago lanceolata L. 1. A growth chamber experiment to examine pre- and postzygotic temperature effects. Evolution 50:865-878.

Lacey, E. P., and D. Herr. 2000. Parental effects in Plantago lanceolata L. III. Measuring parental temperature effects in the field. Evolution 54:1207-1217.

- 2005. Phenotypic plasticity, parental effects and parental care in plants? I. An examination of spike reflectance in Plantago lanceolata (Plantaginaceae). American Journal of Botany 92:920-930.

Lacey, E. P., M. B. Lovin, S. J. Richter, and D. A. Herington. 2010. Floral reflectance, color, and thermoregulation: what really explains geographic variation in thermal acclimation ability of ectotherms? American Naturalist 175:335-349.

Lacey, E. P., D. A. Roach, D. Herr, S. Kincaid, and R. Perrott. 2003. Multigenerational effects of flowering and fruiting phenology in Plantago lanceolata. Ecology 84:2462-2475.

Lande, R. 1982. A quantitative genetic theory of life-history evolution. Ecology 63:607-615.

Lande, R., and S. J. Arnold. 1983. The measurement of selection on correlated characters. Evolution 37:1210-1226.

Lesica, P., and P. M. Kittelson. 2010. Precipitation and temperature are associated with advanced flowering phenology in a semi-arid grassland. Journal of Arid Environments 74:1013-1017.

Levins, R. 1968. Evolution in changing environments. Princeton University Press, Princeton, NJ.

Loeschcke, V., and A. A. Hoffmann. 2007. Consequences of heat hardening on a field fitness component in Drosophila depend on environmental temperature. American Naturalist 169:175-183.

Mitchell-Olds, T., and R. G. Shaw. 1987. Regression analysis of natural selection: statistical inference and biological interpretation. Evolution 41:1149-1161.

Moran, N. A. 1992. The evolutionary maintenance of alternative phenotypes. American Naturalist 139:971-989.

O'Neil, P. 1997. Natural selection on genetically correlated phenological characters in Lythrum salicaria L. (Lythraceae). Evolution 51:267-274.

Parmesan, C. 2006. Ecological and evolutionary responses to recent 
climate change. Annual Review of Ecology, Evolution, and Systematics 37:637-669.

Ragland, G. J., and J. G. Kingsolver. 2008. Evolution of thermotolerance in seasonal environments: the effects of annual temperature variation and life-history timing in Wyeomyia smithii. Evolution 62:1345-1357.

Rathcke, B., and E. P. Lacey. 1985. Phenological patterns of terrestrial plants. Annual Review of Ecology and Systematics 16:179-214.

Robinet, C., and A. Roques. 2010. Direct impacts of recent climate warming on insect populations. Integrative Zoology 5:132-142.

Sherry, R. A., X. Zhou, S. Gu, J. A. Arnone III, D. S. Schimel, P. S. Verburg, L. L. Wallace, and Y. Luo. 2007. Divergence of reproductive phenology under climate warming. Proceedings of the National Academy of Sciences of the USA 104:198-202.

Stiles, E. A., N. B. Cech, S. M. Dee, and E. P. Lacey. 2007. Temperature-sensitive anthocyanin production in flowers of Plantago lanceolata. Physiologia Plantarum 129:756-765.

Stinchcombe, J. R., A. F. Agrawal, P. A. Hohenlohe, S. J. Arnold, and
M. W. Blows. 2008. Estimating nonlinear selection gradients using quadratic regression coefficients: double or nothing? Evolution 62: 2435-2440.

Todd, B. D., D. E. Scott, J. H. K. Pechmann, and J. W. Gibbons. 2011. Climate change correlates with rapid delays and advancements in reproductive timing in an amphibian community. Philosophical Transactions of the Royal Society B: Biological Sciences 278:2191-2197.

Wilczek, A. M., L. T. Burghardt, A. R. Cobb, M. D. Cooper, S. M. Welch, and J. Schmitt. 2010. Genetic and physiological bases for phenological responses to current and predicted climates. Philosophical Transactions of the Royal Society B: Biological Sciences 365:3129-3147.

Associate Editor: Charles F. Baer Editor: Ruth G. Shaw

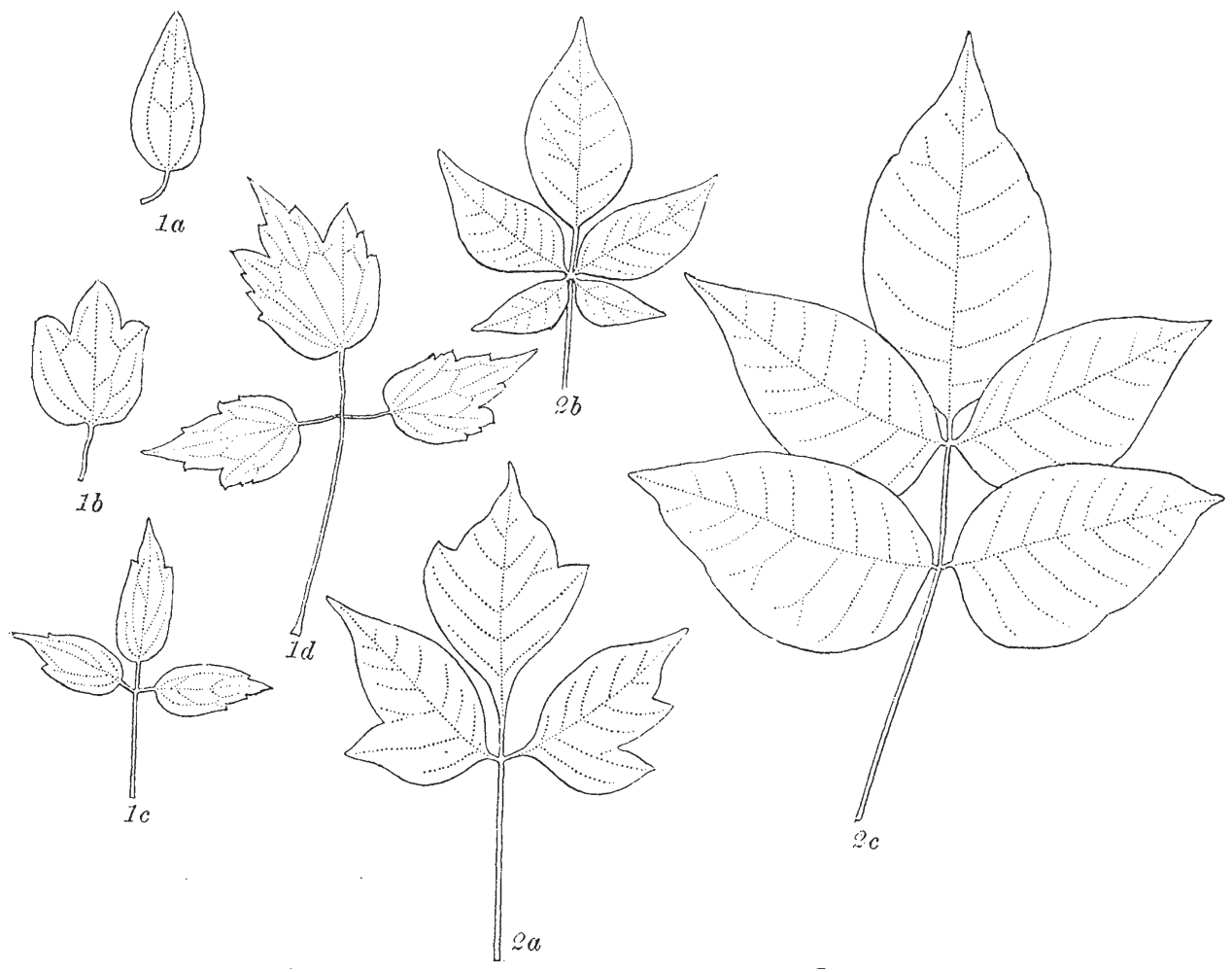

"The simplest form of compound leaf is three-parted or ternate, and is produced by the lateral lobation of a simple leaf. The stages in this process as seen in the mature leaves of Clematis virginiana are shown in figs. $1 a-1 d \ldots .$. In the poison ivy, Rhus toxicodendron, simple leaves are occasionally found, but the typical form is ternate. Many leaves exhibit both basipetal and basifugal features (fig. 2a), and explain the occurrence of both palmate and pinnate leaves in this species (figs. $2 b$ and 2c)." From "A Further Study of Leaf Development" by Frederic T. Lewis (American Naturalist, 1907, 41:701-709). 